\title{
Trends and predictors in methadone maintenance treatment dropout in Shanghai, China: 2005-2011
}

Jinma Ren, $\mathrm{PhD}^{\mathrm{a}, \dagger}$, Zhen Ning, MD, MPH ${ }^{\mathrm{b}, \dagger}$, Carl V. Asche, PhD, MBA ${ }^{\mathrm{a}, \mathrm{c}}$, Minghua Zhuang ${ }^{\mathrm{b}}$, Carmen S. Kirkness, PT, $\mathrm{PhD}^{\mathrm{a}}$, Xiangyang $\mathrm{Ye}^{\mathrm{d}}$, Jie Fu ${ }^{\mathrm{b}}$, Qichao Pan, $\mathrm{MD}^{\mathrm{b}}$

${ }^{a}$ Center for Health Outcomes Research, University of Illinois College of Medicine at Peoria, Illinois, USA

${ }^{\mathrm{b}}$ Shanghai Municipal Center for Disease Control and Prevention, Shanghai, China

${ }^{\mathrm{c}}$ University of Illinois College of Pharmacy at Chicago, Illinois, USA

${ }^{\mathrm{d}}$ Pharmacotherapy Outcomes Research Center, University of Utah College of Pharmacy, Salt Lake City, Utah, USA

Corresponding authors:

(1) Qichao Pan, MD, Shanghai Municipal Center for Disease Control and Prevention, 1380 West Zhongshan Rd, Shanghai 200336, China. Email: qcpan@ @scdc.sh.cn.

(2) Jinma Ren, PhD, Center for Health Outcomes Research, University of Illinois College of Medicine at Peoria, One Illini Drive Box 1649, Peoria, IL 61656, USA. Email: jinmaren@uic.edu.

${ }^{\dagger}$ These authors contributed equally to this work

Paper word count (excluding title, abstract, references, tables, and figures): 3083

Abstract word count: 286 (excluding keywords)

Number of Tables: 3

Number of Figures: 3 


\begin{abstract}
Background: The methadone maintenance treatment (MMT) program has been implemented in Shanghai since 2005. This study aims to portray the trend of MMT dropout and identify predictive factors that may influence dropout in Shanghai MMT clinics, which could assist in the intervention strategy development. Methods: A retrospective evaluation was used in the Shanghai component of the National MMT data management system between January 1, 2005 and December 31, 2011. Cox model for recurrence events was employed to estimate hazard ratio (HR) predicting dropout during the follow-up period. Results: Of all 6169 participants, 63\% dropped out of the program at least once (ranging from 0 to 10 times), and $74 \%$ of them did not return by the end of this study. The average monthly incidence rate of dropout was $4.4 \%$ with a range from 0 to $9.3 \%$. Adjusted analyses demonstrated that the individuals with methadone tapering didn't have a greater probability of dropping out compared to those with stable dosage (HR=1.07, 95\%CI: $0.90-1.27)$. However, there was a higher dropout rate among younger individuals ( $<30$ years vs. $\geq 50$ years old; $\mathrm{HR}=1.41,95 \% \mathrm{CI}$ : 1.16-1.71), among those who were less educated (HR=1.48, 95\%CI: 1.17-1.87), among those who shared needles with others (HR=1.29, 95\%CI: 1.06-1.58), among those whose urine tested positive for opiates ( $\mathrm{HR}=1.69,95 \% \mathrm{CI}$ : 1.51-1.89), and among those who had a low average methadone dose at the initial stable stage of treatment $(\leq 35 \mathrm{mg} /$ day vs. $>65 \mathrm{mg} /$ day; HR $=1.39$, 95\% CI: 1.19-1.63). Conclusions: Shanghai has been facing the challenge of keeping a high MMT retention rate. Methadone tapering after the stable treatment stage with sufficient dosage could be attempted to popularize in the MMT program, aside from considering comprehensive interventions among specific populations, such as the young, poorly educated, opiate-positive and needle sharing individuals.
\end{abstract}

Key words Methadone maintenance treatment; Dropout; Tapering off; Trend; Predictors; Survival analysis 


\section{Introduction}

Opioid dependence has led to diverse social and health problems, such as poverty, violence, criminal behavior, discrimination and sexual transmitted disease epidemic. Due to the transmission of human immunodeficiency virus (HIV) and hepatitis $\mathrm{C}$ virus (HCV), the opioid dependent injecting drug user population is at highest risk of health problems. ${ }^{1,2}$ In China, injecting drug use was still the second single cause of HIV transmission although sexual transmission has become the primary mode of transmission since $2007 .{ }^{3}$ Up to 2010 , over 1.4 million drug users were registered and the number is expected to grow annually; ${ }^{4}$ furthermore, some studies report the prevalence of HIV and HCV among heroin addicts were $1.1 \%$ and $53.9 \%$, respectively. ${ }^{5,6}$ As of 2011 , over three quarters of a million people with HIV infection were estimated among adults 15-49 years of age. ${ }^{3}$ More than 30 million HCV infected individuals were estimated in 1990s, and 17,632 new HCV infections were reported in 2011.,8

Methadone maintenance treatment (MMT) is one of the effective treatments for opiate addiction. ${ }^{9-11}$ Previous studies have established that MMT programs reduce drug use, drug injecting behaviors, drugrelated criminal behaviors, HIV infections, and improve relationships within families of heroin users. ${ }^{12} \mathrm{~A}$ systematic review indicated that opiate substitution treatment was associated with a $54 \%$ reduction in risk of HIV infection among people who inject drugs. ${ }^{13}$ Therefore, eight MMT programs were pilot tested in five provinces within China in 2004 in order to reduce the opiate addiction rates and HIV epidemic health consequences attributed to addiction. ${ }^{14}$ Within six years, the MMT program expanded into a nationwide program involving more than 701 clinics serving approximately 295,000 participants. ${ }^{14,15}$

The effectiveness of MMT is dependent upon timely entry into treatment, adequate medication dose, duration and continuity of treatment, and accompanying medical and psychosocial services. ${ }^{16}$ In order to effectively guide and manage the vast number of MMT clinics in the country, the Ministry of Health, the Ministry of Public Security and the State Food and Drug Administration jointly issued the Temporary Scheme for Community-based Drug Maintenance Treatment for Heroin Dependents in 2003, which was revised into the Implementation Protocol for Community-Based Methadone Maintenance Treatment for Opiate Addicts in 2006. ${ }^{14}$ However, many MMT clinics in China still experience high dropout and relapse rates. ${ }^{17}$ In Guangzhou, of those who are newly admitted MMT clients, $62 \%$ have exhibited poor adherence within the first 6 months since their admission. ${ }^{18}$

Shanghai, an economic center in the mainland of China, had a population of over 23 million in $2010 .{ }^{19}$ Owing to the challenges of controlling drug use and the HIV epidemic in this densely populated metropolis, 14 new MMT clinics were opened in Shanghai during the period of 2005-2009. However, little is known about the MMT compliance and retention in the Shanghai MMT clinics, limiting the development of valid management and intervention strategies. Therefore, the aim of this prospective study was to describe the trend of MMT dropout for those in Shanghai MMT clinics and identify predictive factors that may influence patient retention in Shanghai.

\section{Methods}

\subsection{Study design}

The study consists of an analysis of routinely collected data on all MMT users ( $n=6169$ ) who came from the Shanghai component of the National MMT data management system between January 1, 2005 
and December 31, 2011. ${ }^{20}$ The cohort was used to observe the trend of enrollment and retention in MMT clinics over time. In order to estimate the hazard ratio for dropping out at differing time-points, and to analyze the predictive factors, a sub-cohort of individuals was defined to ensure there was at least three years of follow-up time after the initial evaluation. The sub-cohort $(n=2463)$ included individuals attending MMT clinics between 01/01/2007 to 12/31/2008, Figure 1.

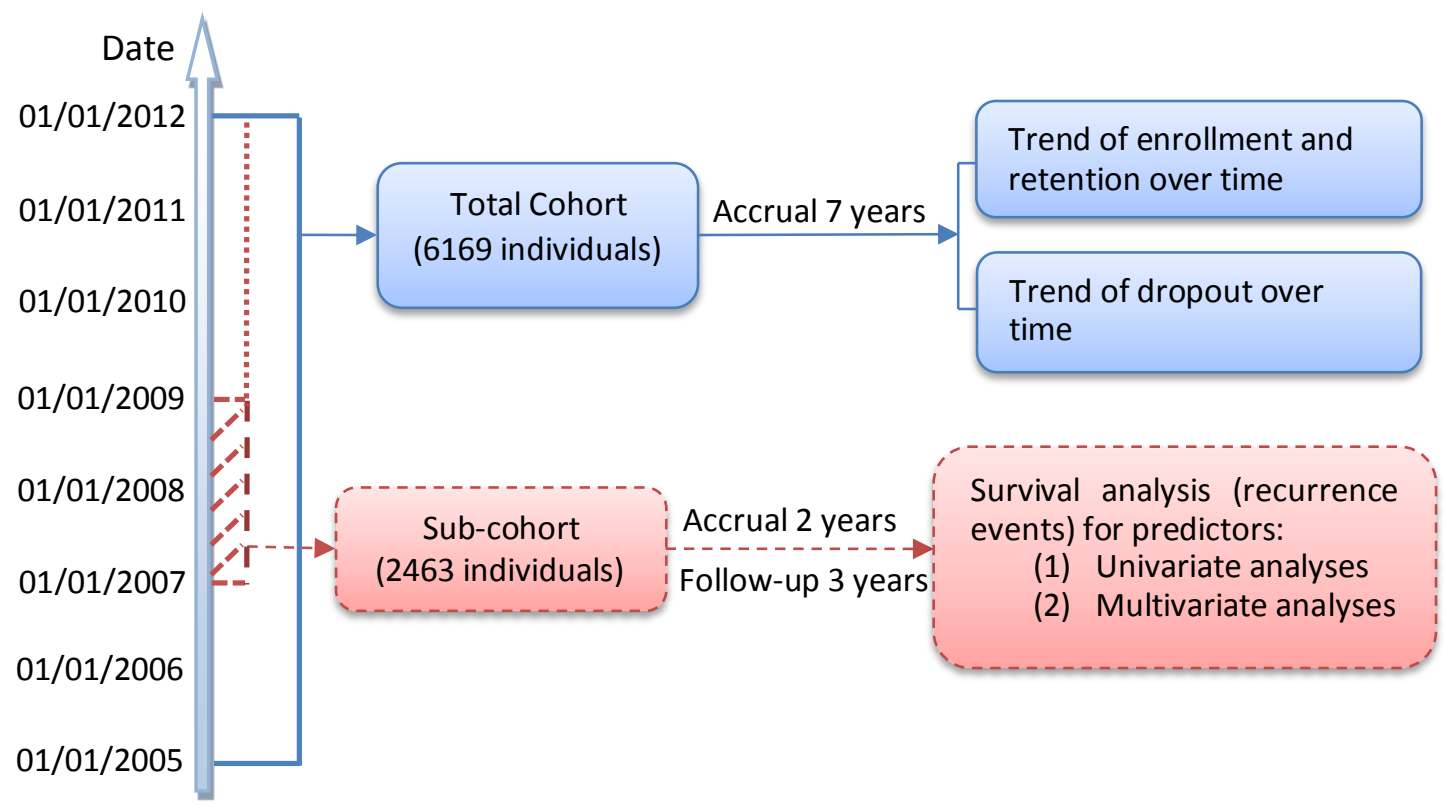

Figure 1 Overview of the prospective cohort study on methadone maintenance treatment

\subsection{Data source and informed consent}

The Shanghai component of the National MMT data management system records contain demographic information at enrollment, history of drug use, laboratory tests, methadone daily doses, reasons for withdrawal, referrals, clinic and physician information. The database is comprised of 8 datasets representing daily dose records, baseline surveys, follow-up surveys, withdrawal reasons, HIV tests, HCV tests, TB (tuberculosis) tests and urine (opiate) tests. There are 2.8 million daily dose records in the dataset. The participants recruited into the MMT included voluntary individuals, those required if released after arrest, and those seeking other medical care. All participants provided informed consent that their medical records could be used for scientific studies when they registered to MMT, and all the data in the study were de-identified.

\subsection{Included and excluded criteria}

Individuals were included in the study if their treatment codes were issued by Shanghai MMT clinics (the first three digits of treatment code were "310") and if their dosing date was during the period of January 1, 2005 to December 31, 2011. The dropout events for each individual were followed over the study period. This study excluded those participants who were not initially registered in Shanghai MMT clinics, and considered their dropout events as missing values if the patients had a permanent referral to other provinces. Finally, there were 6169 individuals and 8398 dropout events in the total cohort. Individuals enrolled during the period of January 1, 2007 to December 31, 2008 established a sub-cohort 
of the study population that had at least three years of available follow-up data. There were 2463 individuals and 2804 dropout events. A few of data entry errors (less than $0.2 \%$ ) about age and dosage were found and corrected after checking with the local MMT clinics.

\subsection{Study measures}

The primary outcome of this study was dropout rate. An event with more than 30 suspended days was considered as one dropout. Suspended days was defined as a period of time that did not have methadone dosing records continuously recorded, except if there was a temporary or permanent referral to other provinces. A patient could have several dropout events. The duration of time (days) that was observed for each event was defined as either the time occurring between the enrollment and the dropout (survival time in survival analysis) or the duration of time occurring between the enrollment and the last date of the study (censor time in survival analysis). The monthly incidence rate of dropout was considered as the number of new dropouts per 100 current individuals in a given month.

Predictor covariates for dropout included age, sex, highest level of education achieved, employed status (yes/no), marital status, clinic (urban/suburban), past drug use history (heroin use, detoxification and needle sharing), most recent urine test (opiate), presence of infectious diseases (HIV/HCV/TB), and two derived variables based upon daily dosage records: 1$)$ average methadone dose ( $\mathrm{mg} / \mathrm{day})$ at the initial stable stage which was defined as 4-6 weeks after enrollment; 2) methadone tapering/increasing that is considered as the average methadone dose (mg/day) after the ninth week decreased/increased by more than $30 \%$ compared to the initial stable stage. See Table 1.

\subsection{Statistical analysis}

Descriptive statistics included mean (standard deviation), frequency and percentage. Multiple imputation (MI procedure) was used to cope with the missing values. Univariate Cox proportional hazards regression models (Cox model) were used to estimate hazard ratio (HR) predicting dropout during the follow-up period, for each variable in Table 1 . All significant $(\mathrm{p}<0.05)$ variables in the univariate analysis were selected as candidates for the forward stepwise multivariate Cox model analysis. Only variables making a statistically significant contribution to the fit of the model were retained for this analysis. A model for recurrent events data was used to conduct univariate and multivariate Cox models (see PHREG example 66.7 in SAS, Time-Dependent Repeated Measurements of a Covariate). All statistics were computed using SAS 9.3 (SAS Institute Inc., Cary, NC, USA).

\section{Results}

\subsection{Participants' characteristics}

From 2005 to 2011, a total 14 MMT clinics were established in Shanghai (1 in May 2005, 4 in September 2006, 3 in August 2007, 4 in September-December 2008 and 2 in October 2009). Of the 14 MMT clinics, seven were considered urban districts (Huangpu, Xuhui, Changning, Putuo, Zhabei, Hongkou and Yangpu), and seven were located in suburban districts (Minhang, Baoshan, Pudong, Songjiang and Qingpu).

As depicted in Table 1, the profiles of individuals representing the sub-cohort (2463 individuals) were very similar with that in the total cohort (6169 individuals). Therefore, the characteristics of individuals 
representing the total cohort only are described here. Among the 6169 individuals, the average age was (40.9 \pm 8.5$)$ years with a range of 18 to 72 years. Almost three-quarters of the population were between 30 and 49 years $(71.8 \%)$. The majority of individuals were male $(77.8 \%)$, Han nationality $(98.4 \%)$ and from the urban clinics $(75.5 \%)$. Only $37.1 \%$ were married and $18.1 \%$ had jobs when they started MMT. The majority of individuals $(65.5 \%)$ were educated at junior high school level or below. The prevalence of HCV, HIV and TB infections at baseline were $68.8 \%, 0.9 \%$ and $0.8 \%$, respectively.

The vast majority of individuals entering MMT were heroin users $(98.9 \%)$, and the injection route was dominant $(67.5 \%)$. Twenty nine percent were opiate-positive through urine test most recently. During the initial stable stage (4-6 weeks), the average methadone dose was $(50.2 \pm 20.5) \mathrm{mg} /$ day per patient, and $21.6 \%$ took the dosage over $65 \mathrm{mg} /$ day. During treatment, $8 \%$ were tapering their methadone dosages. Within 7 days, $94 \%$ had at least one suspension, $36 \%$ had a continuous suspension of $8-14$ days, $21 \%$ had a continuous suspension of 15-30 days, and 62.8\% had at least one dropout (74\% of them did not return by the end of this study period). The average dropout per patient was one $(0.87 \pm 0.91$, median was 1$)$, ranging from 0 to 10 dropouts.

Table 1 Profiles of the participants of methadone maintenance treatment in Shanghai

\begin{tabular}{|c|c|c|c|c|}
\hline \multirow{2}{*}{ Items } & \multicolumn{2}{|c|}{ Sub-cohort (2007-2008) } & \multicolumn{2}{|c|}{ Total cohort $(2005-2011)$} \\
\hline & Num.(N=2463) & \multirow[t]{2}{*}{ Pct. $(\%)$} & Num. $(\mathrm{N}=6169)$ & \multirow[t]{2}{*}{ Pct. (\%) } \\
\hline Age (year), $\bar{x} \pm s$ & $40.0 \pm 8.2$ & & $40.9 \pm 8.5$ & \\
\hline$<30$ & 343 & 13.9 & 761 & 12.3 \\
\hline 30-39 & 758 & 30.8 & 1805 & 29.3 \\
\hline $40-49$ & 1075 & 43.7 & 2622 & 42.5 \\
\hline$\geq \mathbf{5 0}$ & 287 & 11.6 & 981 & 15.9 \\
\hline \multicolumn{5}{|l|}{ Gender } \\
\hline Female & 615 & 25.0 & 1370 & 22.2 \\
\hline Male & 1848 & 75.0 & 4799 & 77.8 \\
\hline \multicolumn{5}{|l|}{ Marital status } \\
\hline Single & 963 & 39.1 & 2462 & 39.9 \\
\hline Married & 975 & 39.6 & 2287 & 37.1 \\
\hline Divorced & 506 & 20.5 & 1360 & 22.0 \\
\hline Others(widow) & 19 & 0.8 & 60 & 1.0 \\
\hline \multicolumn{5}{|l|}{ Ethnic nationality } \\
\hline Han & 2420 & 98.3 & 6072 & 98.4 \\
\hline Others & 43 & 1.7 & 97 & 1.6 \\
\hline \multicolumn{5}{|l|}{ Clinic } \\
\hline Urban & 1937 & 78.7 & 4659 & 75.5 \\
\hline Suburban & 526 & 21.4 & 1510 & 24.5 \\
\hline \multicolumn{5}{|l|}{ Employed } \\
\hline No & 2025 & 82.2 & 5053 & 81.9 \\
\hline Yes & 438 & 17.8 & 1116 & 18.1 \\
\hline \multicolumn{5}{|l|}{ Education } \\
\hline College and above & 74 & 3.0 & 165 & 2.7 \\
\hline Senior high school & 823 & 33.4 & 1963 & 31.8 \\
\hline Junior high school & 1467 & 59.6 & 3757 & 60.9 \\
\hline Elementary and below & 99 & 4.0 & 284 & 4.6 \\
\hline \multicolumn{5}{|l|}{ Detoxification history } \\
\hline No & 318 & 12.9 & 736 & 11.9 \\
\hline Yes & 2145 & 87.1 & 5433 & 88.1 \\
\hline \multicolumn{5}{|l|}{ Heroin use at registration } \\
\hline 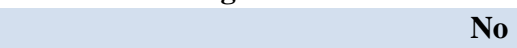 & 38 & 1.5 & 69 & 1.1 \\
\hline Yes & 2425 & 98.5 & 6100 & 98.9 \\
\hline
\end{tabular}




\begin{tabular}{|c|c|c|c|c|}
\hline \multicolumn{5}{|l|}{ Drug-use route } \\
\hline Only injection & 1618 & 65.7 & 3893 & 63.1 \\
\hline Injection and oral & 69 & 2.8 & 271 & 4.4 \\
\hline Only oral & 776 & 31.5 & 2005 & 32.5 \\
\hline \multicolumn{5}{|l|}{ Needle sharing history (lifetime) } \\
\hline No & 2330 & 94.6 & 5699 & 92.4 \\
\hline Yes & 133 & 5.4 & 470 & 7.6 \\
\hline \multicolumn{5}{|l|}{ Presence of $\mathrm{HCV}$ infection } \\
\hline$e_{-1}$ & 673 & 33.7 & 1572 & 31.2 \\
\hline Yes & 1324 & 66.3 & 3462 & 68.8 \\
\hline Missing $^{1}$ & 466 & & 1135 & \\
\hline \multicolumn{5}{|l|}{ Presence of HIV infection } \\
\hline No & 1828 & 99.5 & 4812 & 99.1 \\
\hline Yes & 10 & 0.5 & 42 & 0.9 \\
\hline Missing $^{1}$ & 625 & & 1315 & \\
\hline \multicolumn{5}{|l|}{ Presence of TB infection } \\
\hline 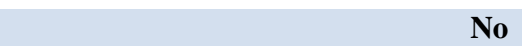 & 833 & 99.8 & 1913 & 99.2 \\
\hline Yes & 2 & 0.2 & 15 & 0.8 \\
\hline Missing $^{1}$ & 1628 & & 4241 & \\
\hline \multicolumn{5}{|l|}{ Most recent urine test (opiate) } \\
\hline Positive & 647 & 27.5 & 1711 & 28.8 \\
\hline Negative & 1708 & 72.5 & 4238 & 71.2 \\
\hline Missing $^{1}$ & 108 & & 220 & \\
\hline $\begin{array}{l}\text { Average methadone dose at the } \\
\text { initial stable stage }(\mathrm{mg} / \mathrm{day}), \bar{x} \pm s\end{array}$ & $50.7 \pm 20.6$ & & $50.2 \pm 20.5$ & \\
\hline$\leq 35$ & 572 & 25.9 & 1467 & 26.1 \\
\hline $36-50$ & 653 & 29.5 & 1716 & 30.6 \\
\hline $51-65$ & 463 & 20.9 & 1219 & 21.7 \\
\hline$>65$ & 524 & 23.7 & 1210 & 21.6 \\
\hline Missing $^{2}$ & 251 & & 557 & \\
\hline \multicolumn{5}{|l|}{ Methadone dose change over time } \\
\hline Stable & 1343 & 54.5 & 3543 & 57.4 \\
\hline Tapering & 217 & 8.8 & 498 & 8.1 \\
\hline Increasing & 440 & 17.9 & 1035 & 16.8 \\
\hline Unknown $^{3}$ & 463 & 18.8 & 1093 & 17.7 \\
\hline
\end{tabular}

\subsection{Trend of dropout rate}

Since the end of 2006, the number of enrolled individuals increased exponentially, especially during the period from 2006 to 2008. At the end of 2011, 6169 individuals had been cumulatively enrolled, however, only 61.3\% (3063/6169) were still retained (regularly visiting MMT clinics). As shown in Figure 2, there was no clear periodic change in the dropout rate. However, the dropout rate was notably higher during the period from 2006 to 2008 coupled with a high enrolment rate, and became lower and more stable after 2009. The average monthly incidence of dropout was $4.4 \%$ with a range from 0 to $9.3 \%$.

Overall, the participants with methadone tapering had similar dropout rates with those who kept stable doses from 2005 to 2011, and even had lower dropout rates at some particular time points (Figure 3). 


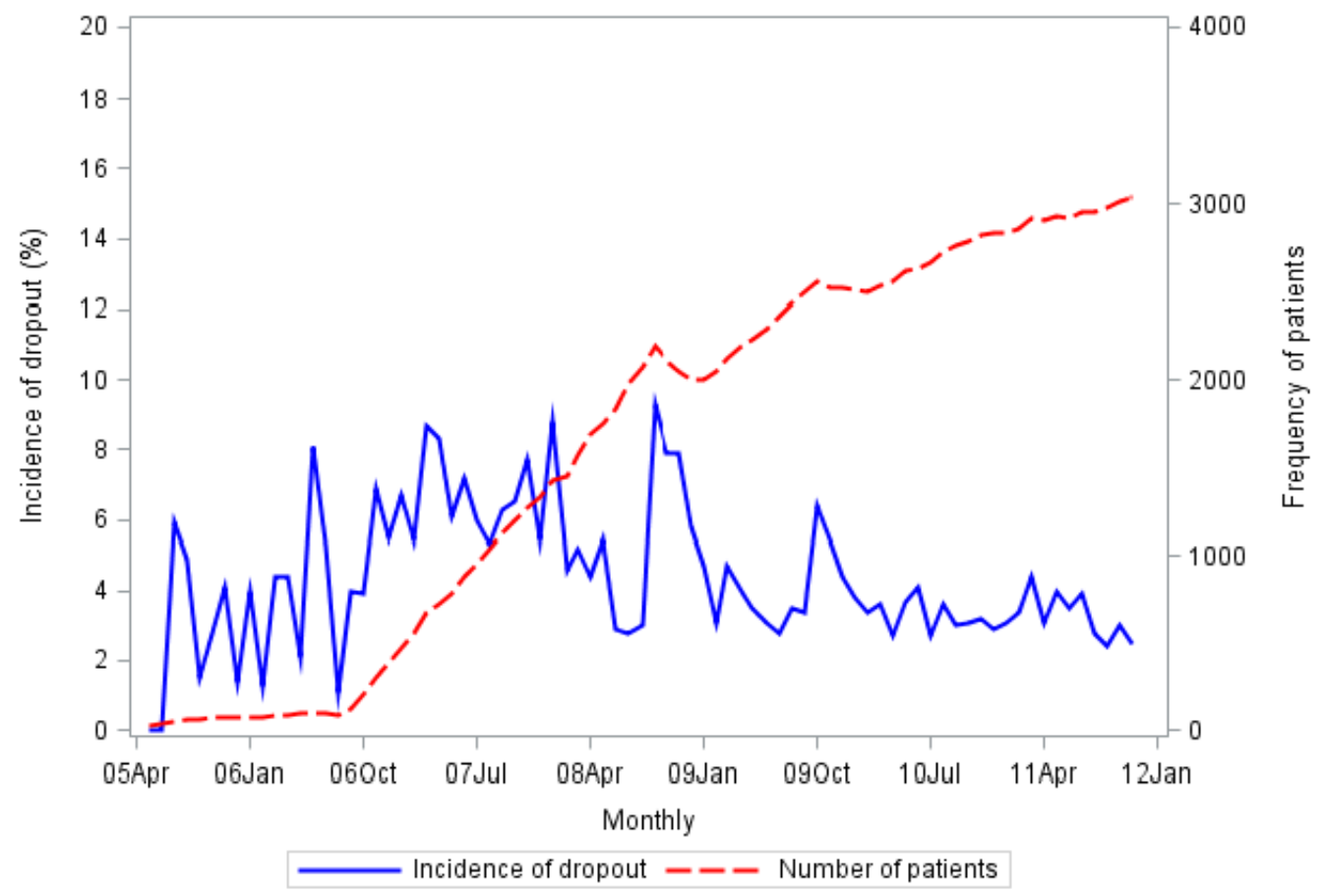

Figure 2 Trend of monthly dropout in methadone maintenance treatment in Shanghai 2005-2011

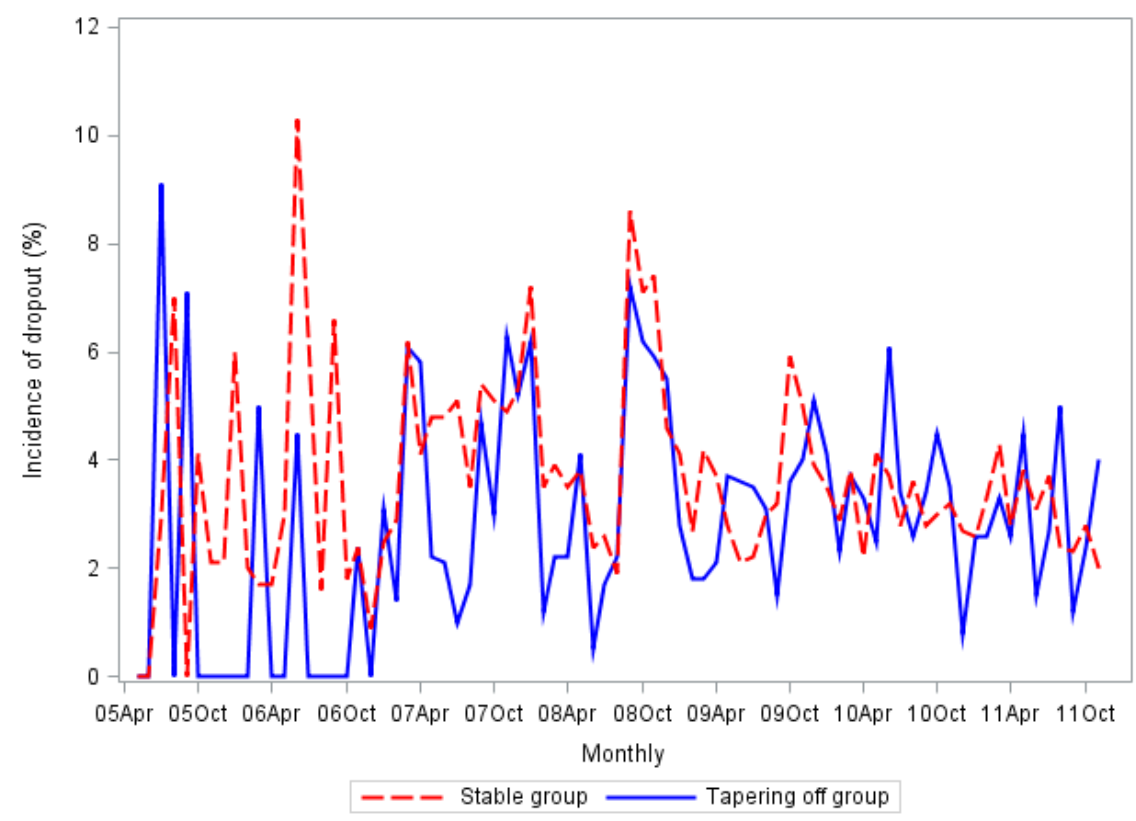

Figure 3 Comparison of monthly dropout rate between methadone tapering and stable groups 


\subsection{Dropout predictors and multivariate analysis}

The survival analyses were only conducted using the individuals represented in the sub-cohort (2463 individuals, 2804 events). As depicted in Table 2, the univariate cox model analysis demonstrated that nine candidate factors could be associated with dropout $(P<0.05)$. Then, our multivariate stepwise Cox regression model involved the nine candidate factors. Finally, only six predictors (age, education, needle sharing, urine opiate test, average methadone dose at the stable stage, and methadone dose change over time) were selected into the final Cox model, however, marital status, drug-use route and HCV infection were excluded because their contributions were not significant any more $(P>0.05)$.

As shown in Table 3, the individuals with methadone tapering didn't have a greater probability of dropping out compared to those with stable dosage (HR=1.07, 95\%CI: 0.90-1.27). However, there was a higher dropout rate among younger individuals ( $<30$ years vs. $\geq 50$ years old; HR=1.41, 95\%CI: 1.16-1.71), among those who were less educated $(\mathrm{HR}=1.48$, 95\%CI: 1.17-1.87), among those who shared needles with others (HR=1.29, 95\%CI: 1.06-1.58), among those whose urine tested positive for opiates (HR=1.69, 95\%CI: 1.51-1.89), and among those who had a low average methadone dose at the initial stable stage of treatment $(\leq 35 \mathrm{mg} /$ day vs. $>65 \mathrm{mg} /$ day; $\mathrm{HR}=1.39,95 \%$ CI: $1.19-1.63)$. 
Table 2 Univariate Cox model analysis with hazard ratios for dropout from methadone maintenance treatment

\begin{tabular}{|c|c|c|c|}
\hline Factors & $\begin{array}{r}\text { Hazard } \\
\text { Ratio }\end{array}$ & $95 \% \mathrm{CI}$ & $P$ value \\
\hline \multicolumn{4}{|l|}{ Age (ref: $\geq 50$ years) } \\
\hline$<30$ & 1.53 & $1.26-1.84$ & $<0.001$ \\
\hline $30-39$ & 1.42 & $1.20-1.68$ & $<0.001$ \\
\hline $40-49$ & 1.17 & $0.99-1.38$ & 0.061 \\
\hline \multicolumn{4}{|l|}{ Gender (ref: Male) } \\
\hline Female & 0.93 & $0.84-1.04$ & 0.209 \\
\hline \multicolumn{4}{|l|}{ Marital status (ref: Married) } \\
\hline Single & 1.14 & $1.03-1.26$ & 0.014 \\
\hline Divorced and others & 0.96 & $0.85-1.09$ & 0.518 \\
\hline \multicolumn{4}{|l|}{ Clinic (ref: Urban) } \\
\hline Suburban & 1.04 & $0.93-1.17$ & 0.450 \\
\hline \multicolumn{4}{|l|}{$\begin{array}{l}\text { Education (ref: senior high school and } \\
\text { above) }\end{array}$} \\
\hline Elementary \& below & 1.34 & $1.07-1.67$ & 0.010 \\
\hline Junior high school & 1.00 & $0.90-1.10$ & 0.920 \\
\hline \multicolumn{4}{|l|}{ Employed (ref: No) } \\
\hline Yes & 1.12 & $0.99-1.26$ & 0.074 \\
\hline \multicolumn{4}{|l|}{ Detoxification history (ref: No) } \\
\hline - & 0.96 & $0.84-1.11$ & 0.598 \\
\hline \multicolumn{4}{|l|}{ Drug-use route (ref: Oral) } \\
\hline Injection & 1.13 & $1.02-1.25$ & 0.021 \\
\hline \multicolumn{4}{|l|}{ Needle sharing (ref: No) } \\
\hline Yes & 1.40 & $1.16-1.69$ & 0.001 \\
\hline \multicolumn{4}{|l|}{ HCV (ref: Negative) } \\
\hline Positive & 0.89 & $0.80-0.98$ & 0.019 \\
\hline \multicolumn{4}{|l|}{ Urine test (ref: Opiate-negative) } \\
\hline Positive & 2.46 & $2.23-2.71$ & $<0.001$ \\
\hline \multicolumn{4}{|l|}{$\begin{array}{l}\text { Average methadone dose at the stable } \\
\text { stage (ref: }>65 \mathrm{mg} / \text { day) }\end{array}$} \\
\hline$\leq 35$ & 1.37 & $1.19-1.57$ & $<0.001$ \\
\hline $36-50$ & 1.54 & $1.35-1.76$ & $<0.001$ \\
\hline $51-65$ & 1.52 & $1.32-1.75$ & $<0.001$ \\
\hline \multicolumn{4}{|l|}{$\begin{array}{l}\text { Methadone dose change over time (ref: } \\
\text { Stable) }\end{array}$} \\
\hline Tapering & 0.98 & $0.83-1.15$ & 0.77 \\
\hline Increasing & 0.65 & $0.56-0.74$ & $<0.001$ \\
\hline Unknown & 17.74 & $15.70-20.50$ & $<0.001$ \\
\hline
\end{tabular}


Table 3 Multivariate stepwise Cox model analysis with hazard ratios for dropout from methadone maintenance treatment

\begin{tabular}{|c|c|c|c|}
\hline Factors & $\begin{array}{r}\text { Hazard } \\
\text { Ratio }\end{array}$ & $95 \% \mathrm{CI}$ & $P$ value \\
\hline \multicolumn{4}{|l|}{ Age (ref: $\geq 50$ years) } \\
\hline$<30$ & 1.41 & $1.16-1.71$ & 0.001 \\
\hline $30-39$ & 1.37 & $1.15-1.63$ & $<0.001$ \\
\hline $40-49$ & 1.15 & $0.98-1.37$ & 0.095 \\
\hline \multicolumn{4}{|l|}{ Education (ref: Senior high school and more) } \\
\hline Elementary \& below & 1.48 & $1.17-1.87$ & 0.001 \\
\hline Junior high school & 1.00 & $0.90-1.10$ & 0.925 \\
\hline \multicolumn{4}{|l|}{ Needle sharing (ref: No) } \\
\hline Yes & 1.29 & $1.06-1.58$ & 0.012 \\
\hline \multicolumn{4}{|l|}{ Urine test (ref: Opiate-negative) } \\
\hline Positive & 1.69 & $1.51-1.89$ & $<0.001$ \\
\hline \multicolumn{4}{|l|}{$\begin{array}{l}\text { Average methadone dose at the stable } \\
\text { stage (ref: }>65 \mathrm{mg} / \text { day) }\end{array}$} \\
\hline$\leq 35$ & 1.39 & $1.19-1.63$ & $<0.001$ \\
\hline $36-50$ & 1.36 & $1.18-1.56$ & $<0.001$ \\
\hline $51-65$ & 1.20 & $1.03-1.39$ & 0.016 \\
\hline \multicolumn{4}{|l|}{$\begin{array}{l}\text { Methadone dose change over time (ref: } \\
\text { Stable) }\end{array}$} \\
\hline Tapering & 1.07 & $0.90-1.27$ & 0.448 \\
\hline Increasing & 0.57 & $0.49-0.67$ & $<0.001$ \\
\hline Unknown & 12.77 & $11.09-14.71$ & $<0.001$ \\
\hline
\end{tabular}

\section{Discussion}

Although the MMT program has been implemented in Shanghai since 2005, our study is the first to comprehensively report the longitudinal trends and predictors of dropout of individuals who attend the MMT clinics for treatment. This study found that both the rates of increasing enrollment and dropout have slowed since 2009. This may in part be due to the health authority and other governmental departments realizing that 14 clinics had satisfied the need in Shanghai and therefore no new MMT clinics were opened. In order to reduce the harm of drug use and to further control the HIV epidemic, the Chinese government has invested heavily in human resources and funds to run and manage the MMT programs. High dropout and relapse rates, however, have continued to be tough issues that challenge Chinese MMT clinics. ${ }^{17,18}$ In this study, $62.8 \%$ of individuals had dropped out, and only $61.3 \%$ were finally retained up until the end of 2011. Six factors including age, education, needle sharing, opiate urine test, average methadone dosage at the initial stable stage, and methadone dose change over time (tapering, stable or increasing), were found to be significantly associated with dropout from MMT according to adjusted multivariate analyses.

Prior studies have not demonstrated that age is an important predictor in MMT clinic dropout rates among other provinces of China, ${ }^{18,21}$ which may be attributed to inappropriate age grouping, such as 3661 years or over 40 years. In our study, two age groups (less than 30years, 30-39 years) were found to have higher likelihood for dropping out compared to the older group (over 50 years). It is well know that older drug users often age out of high intensity use and that younger individuals typically have riskier 
profiles which interfere with their respective adherence to MMT. In addition, those with low levels of education may have poor understanding and acceptance of rules and regulations, and also reflect lower socio-economic status and other living conditions, so that they could be more likely to drop out from the MMT program.

Many studies have illustrated that higher methadone dose is associated with individuals remaining in treatment longer. ${ }^{22-26}$ This study also found that individuals that had low methadone doses (less than $65 \mathrm{mg} /$ day) during the initial stable stage, were more likely to drop out from MMT clinic as compared to those that had methadone doses of $>65 \mathrm{mg} / \mathrm{day}$. Also, individuals with methadone increasing over time were less likely to dropout. However, timelessly increasing methadone dosage is not an optimal approach to prevent them from dropping out because it is not feasible and it could drive them to addict methadone. Fortunately, it was surprising to find that individuals with methadone tapering didn't have a higher probability of dropping out than those with stable dosage. In China, some of the service providers and individuals express concerns that methadone is 'yet another drug' and often request reduced dosages to avoid methadone addiction. ${ }^{27,28}$ It is true that "tapering off" methadone has been proposed in recent studies. ${ }^{29,30}$ But, people must be clearly aware of what is the appropriate stage for methadone tapering. Sufficient methadone dosage should be provided at the initial stable stage after the first two or three weeks adjusting stage. Then, tapering off methadone could be served to become an attempt among qualified individuals, which might benefit both participants and governments through saving the health resources. Therefore, it could be concluded that proper dosages prescribed at different stages would be crucial for MMT retention.

In our study, 29\% of individuals were found to have positive-opiate at most recent urine tests. These individuals were more likely to dropout from MMT treatment as compared to those with negative-opiate urine tests ( $\mathrm{HR}=1.66,95 \%$ : 1.49-1.86). Also, individuals who shared needles with other injection drug users had a higher dropout hazard ratio compared to other individuals. Our findings support previous literature that the risk of early dropout among individuals living with other drug users was 2.71 times than other individuals in an MMT program. ${ }^{21}$ This study hypothesized that the addiction of these individuals was more serious and their relapse to drug use would cause a dropout because the relapse was a disruption to the MMT guideline. On the other hand, it could also reflect methadone doses were too low and they were taking other opiates to medicate withdrawal symptoms. Clearly, it is necessary to pay more attention to the risk factors experienced by those that access MMT and to the possible triggers different contexts and environments may produce.

The success of the MMT program can be partially attributed to the individual adherence to the daily treatment. The vast majority of individuals in this study suspended their treatments. Furthermore, some individuals had multiple repeated instances of dropout. The high proportion of people that suspend their daily methadone dose required guidelines to be revised to evaluate if different MMT clinic guidelines would decrease the frequency and duration of suspensions. According to the Chinese management guideline of MMT clinics: individuals must visit specific clinics every day and intake methadone at the sites; an extra prescription for the next day is prohibited; a small fee $(\$ 1.5)$ is needed for methadone per day; and individuals with more than 7 continuously suspended days need to re-enroll. It is thought, that providing an extra prescription to the patient and allowing methadone doses to be carried out of clinics were permissible, some individuals would not consume their medicine but rather sell them in the market. 
Therefore, it becomes being recognized that some individuals could cease treatment either temporarily or permanently as they could not tolerate these rigorous rules while strict management is implemented.

This is a valuable study using the long-term follow-up data based on the national MMT management system in China. However, there are limitations to consider when the results are interpreted. First, the dropout was defined as no-show (without dosing record) for 30 days continuously, which could misclassify individuals suspended temporarily into the dropout group, and vice versa. Second, relationship of family members, social network and psychological issues were not captured in the database. Third, it also should exercise caution in extrapolating the results of this study to MMT population in other areas because the characteristics of MMT population could be different.

Overall, MMT clinics have played an important role in controlling the HIV/HCV pandemic and drug abuse in China, ${ }^{31}$ but it is still a challenge to maintain a high MMT retention rate in Shanghai. Methadone tapering after the stable treatment stage with sufficient dosage could be attempted to popularize in MMT, aside from that comprehensive interventions including medical treatment, behavior education, psychological care, social and family support should be considered to address this issue, especially among specific populations, such as the young, poorly educated, opiate-positive individuals and injection drug users.

\section{Acknowledgements}

The authors would like to thank the support of Shanghai Municipal Working Group of Methadone Maintenance Treatment, and also thank all staff and individuals of MMT clinics in Shanghai, who contributed to the data in this study.

\section{Reference}

1. Aceijas C, Stimson GV, Hickman M, Rhodes T. Global overview of injecting drug use and HIV infection among injecting drug users. AIDS 2004;18:2295-303.

2. Aceijas $\mathrm{C}$, Rhodes T. Global estimates of prevalence of $\mathrm{HCV}$ infection among injecting drug users. The International journal on drug policy 2007;18:352-8.

3. 2012 China AIDS Response Progress Report. Ministry of Health of the People's Republic of China, 2012. (Accessed January 22, 2013, at http://aidsdatahub.org/en/reference-librarycols2/item/24194ungass-country-progress-report-china-state-council-aids-working-committee-office-china-2012)

4. Over 1.4 million drug users were registered and about 70,000 had not relapsed for over three years. Narcotics Control Bureau of the Ministry of Public Security, 2010. (Accessed January 22, 2013, at http://www.mps.gov.cn/n16/n80209/n80421/2636872.html)

5. Zhan D, Li D. Analysis of HIV, syphilis and HBV infections in sera of 1639 heroin addicts. Chin $\mathbf{J}$ AIDS STD 2003;9:98-9.

6. Huang H, Zhou H, Zheng X. A study on HBV HCV Infection in 2260 Cases with Intravenous Infection Heroin Addicts. Disease Surveillance 2000;15:128-30.

7. The report of national notifiable infectious diseases issued by the Ministry of Health in December 2011. Ministry of Health of the People's Republic of China, 2011. (Accessed December 3, 2012, at http://www.moh.gov.cn/mohjbyfkzj/s3578/201201/53904.shtml)

8. Liu L, Wei L. Epidemiology of hepatitis C virus. Infectious Disease Information 2007;20:261-4.

9. Payte JT. A brief history of methadone in the treatment of opioid dependence: a personal perspective. Journal of psychoactive drugs 1991;23:103-7. 
10. Ward J, Mattick RP, Hall W. The effectiveness of methadone maintenance treatment: an overview. Drug and alcohol review 1994;13:327-35.

11. Haig T. Randomized controlled trial proves effectiveness of methadone maintenance treatment in prison. Canadian HIV/AIDS policy \& law review / Canadian HIV/AIDS Legal Network 2003;8:48.

12. Pang L, Hao Y, Mi G, et al. Effectiveness of first eight methadone maintenance treatment clinics in China. AIDS 2007;21 Suppl 8:S103-7.

13. MacArthur GJ, Minozzi S, Martin N, et al. Opiate substitution treatment and HIV transmission in people who inject drugs: systematic review and meta-analysis. BMJ 2012;345:e5945.

14. Yin W, Hao Y, Sun X, et al. Scaling up the national methadone maintenance treatment program in China: achievements and challenges. International journal of epidemiology 2010;39 Suppl 2:ii29-37.

15. Cao XB, Z.Y. W. Methadone maintenance treatment as an HIV prevention strategy: a research update. Chin J AIDS STD 2011:196-9.

16. WHO, UNODC, UNAIDS. Substitution maintenance therapy in the management of opioid dependence and HIV/AIDS prevention: position paper. Geneva: WHO; 2004.

17. Qian HZ, Schumacher JE, Chen HT, Ruan YH. Injection drug use and HIV/AIDS in China: review of current situation, prevention and policy implications. Harm reduction journal 2006;3:4.

18. Gu J, Xu H, Lau JT, et al. Misconceptions predict dropout and poor adherence prospectively among newly admitted first-time methadone maintenance treatment clients in Guangzhou, China. Addiction 2012.

19. Shanghai Statistical Yearbook 2011. Shanghai Municipal Statistics Bureau (Accessed December 16, 2012, at http://www. stats-sh.gov.cn/data/toTinj.xhtml?y=2011e.)

20. Mao Y, Wu Z, Poundstone K, et al. Development of a unified web-based national HIV/AIDS information system in China. International journal of epidemiology 2010;39 Suppl 2:ii79-89.

21. Che Y, Assanangkornchai S, McNeil E, et al. Predictors of early dropout in methadone maintenance treatment program in Yunnan province, China. Drug and alcohol review 2010;29:263-70.

22. Bao YP, Liu ZM, Epstein DH, Du C, Shi J, Lu L. A meta-analysis of retention in methadone maintenance by dose and dosing strategy. The American journal of drug and alcohol abuse 2009;35:28-33.

23. Brady TM, Salvucci S, Sverdlov LS, et al. Methadone dosage and retention: an examination of the 60 $\mathrm{mg}$ /day threshold. Journal of addictive diseases 2005;24:23-47.

24. Magura S, Nwakeze PC, Demsky SY. Pre- and in-treatment predictors of retention in methadone treatment using survival analysis. Addiction 1998;93:51-60.

25. Caplehorn JR, Bell J. Methadone dosage and retention of patients in maintenance treatment. The Medical journal of Australia 1991;154:195-9.

26. Maremmani I, Nardini R, Zolesi O, Castrogiovanni P. Methadone dosages and therapeutic compliance during a methadone maintenance program. Drug and alcohol dependence 1994;34:163-6.

27. Hser YI, Li J, Jiang H, et al. Effects of a randomized contingency management intervention on opiate abstinence and retention in methadone maintenance treatment in China. Addiction 2011;106:1801-9.

28. Wu F, Peng CY, Jiang H, et al. Methadone maintenance treatment in China: perceived challenges from the perspectives of service providers and patients. J Public Health (Oxf) 2012. Epub 2012 Sept 19.

29. Holmes D. Prescription drug addiction: the treatment challenge. Lancet 2012;379:17-8.

30. Calsyn DA, Malcy JA, Saxon AJ. Slow tapering from methadone maintenance in a program encouraging indefinite maintenance. Journal of substance abuse treatment 2006;30:159-63.

31. Wu Z, Sullivan SG, Wang Y, Rotheram-Borus MJ, Detels R. Evolution of China's response to HIV/AIDS. Lancet 2007;369:679-90. 